\title{
Postpartum Blood Loss in Women Treated for Intrahepatic Cholestasis of Pregnancy
}

Furrer, Romana ; Winter, Katharina ; Schäffer, Leonhard ; Zimmermann, Roland ; Burkhardt, Tilo ; Haslinger, Christian

\begin{abstract}
OBJECTIVE To evaluate postpartum blood loss in women with treated intrahepatic cholestasis of pregnancy. METHODS In a retrospective case-control study, 15,083 deliveries including 348 women with intrahepatic cholestasis of pregnancy (2.3\%) were analyzed from 2004 to 2014 . To adjust for differences in baseline characteristics, a propensity analysis was performed and women in the control group were matched to the women in the intrahepatic cholestasis of pregnancy group in a 5:1 ratio. Blood loss was analyzed by estimated blood loss and $\Delta$ hemoglobin ( $\mathrm{Hb}$, difference between prepartum and postpartum $\mathrm{Hb}$ ). A subgroup analysis regarding severity of intrahepatic cholestasis of pregnancy based on maximum bile acid level (mild [less than 40 micromoles/L], moderate [40-99 micromoles/L], and severe intrahepatic cholestasis of pregnancy [100 micromoles/L or greater]) was performed. Differences in estimated blood loss, $\Delta \mathrm{Hb}$, and meconium staining between subgroups were analyzed. A Spearman rank correlation was performed to evaluate the association of bile acid levels and blood loss within subgroups. RESULTS Estimated blood loss (median 400 [300-600] mL compared with 400 [300-600] mL, P=.22), $\Delta \mathrm{Hb}(14.0[5.0-22.0]$ compared with $12.0[4.0-21.0] \mathrm{g} / \mathrm{L}, \mathrm{P}=.09)$, meconium staining (14.5\% compared with $11.4 \%, \mathrm{P}=.12)$, and number of stillbirths after 26 weeks of gestation $(0.6 \%$ compared with $1.8 \%$, $\mathrm{P}=.10)$ were not significantly different in the study compared with the control group. In moderate and severe intrahepatic cholestasis of pregnancy, meconium staining was observed significantly more often compared with that in a control group $(23.0 \%$ and $32.3 \%$ compared with $11.4 \%, \mathrm{P}<.01$ ). There was no correlation between estimated blood loss or $\Delta \mathrm{Hb}$ and severity of intrahepatic cholestasis of pregnancy. CONCLUSIONS In our cohort of women with intrahepatic cholestasis of pregnancy who are treated with ursodeoxycholic acid and have planned delivery (induction of labor or planned cesarean delivery) at 38 weeks of gestation, no differences in postpartum blood loss were seen.
\end{abstract}

DOI: https://doi.org/10.1097/AOG.0000000000001693

Posted at the Zurich Open Repository and Archive, University of Zurich

ZORA URL: https://doi.org/10.5167/uzh-126808

Journal Article

Published Version

Originally published at:

Furrer, Romana; Winter, Katharina; Schäffer, Leonhard; Zimmermann, Roland; Burkhardt, Tilo; Haslinger, Christian (2016). Postpartum Blood Loss in Women Treated for Intrahepatic Cholestasis of Pregnancy.

Obstetrics and Gynecology, 128(5):1048-1052.

DOI: https://doi.org/10.1097/AOG.0000000000001693 


\title{
Postpartum Blood Loss in Women Treated for Intrahepatic Cholestasis of Pregnancy
}

\author{
Romana Furrer, MD, Katharina Winter, Leonhard Schäffer, MD, Roland Zimmermann, MD, \\ Tilo Burkhardt, $M D$, and Christian Haslinger, $M D$
}

OBJECTIVE: To evaluate postpartum blood loss in women with treated intrahepatic cholestasis of pregnancy.

METHODS: In a retrospective case-control study, 15,083 deliveries including 348 women with intrahepatic cholestasis of pregnancy (2.3\%) were analyzed from 2004 to 2014. To adjust for differences in baseline characteristics, a propensity analysis was performed and women in the control group were matched to the women in the intrahepatic cholestasis of pregnancy group in a 5:1 ratio. Blood loss was analyzed by estimated blood loss and $\Delta$ hemoglobin $(\mathrm{Hb}$, difference between prepartum and postpartum $\mathrm{Hb}$ ). A subgroup analysis regarding severity of intrahepatic cholestasis of pregnancy based on maximum bile acid level (mild [less than 40 micromoles/L], moderate [40-99 micromoles/L], and severe intrahepatic cholestasis of pregnancy [100 micromoles/L or greater]) was performed. Differences in estimated blood loss, $\Delta \mathrm{Hb}$, and meconium staining between subgroups were analyzed. A Spearman rank correlation was performed to evaluate the association of bile acid levels and blood loss within subgroups.

RESULTS: Estimated blood loss (median 400 [300-600] $\mathrm{mL}$ compared with 400 [300-600] $\mathrm{mL}, P=.22), \Delta \mathrm{Hb}(14.0$ [5.0-22.0] compared with 12.0 [4.0-21.0] g/L, $P=.09)$, meconium staining $(14.5 \%$ compared with $11.4 \%$, $P=.12$ ), and number of stillbirths after 26 weeks of gestation $(0.6 \%$ compared with $1.8 \%, P=.10)$ were not

From the Division of Obstetrics, University Hospital of Zürich, and the University of Zürich, and the Division of Obstetrics, Kantonsspital Baden, Baden, Switzerland.

Presented as a poster at the 27th German Congress of Perinatal Medicine, December 1-3, 2015, Berlin, Germany.

Corresponding author: Romana Furrer, $M D$, Division of Obstetrics, University Hospital of Zürich, Frauenklinikstrasse 10, 8091 Zürich, Switzerland; e-mail: romana.furrer@usz.ch.

\section{Financial Disclosure}

The authors did not report any potential conflicts of interest.

(C) 2016 by The American College of Obstetricians and Gynecologists. Published by Wolters Kluwer Health, Inc. All rights reserved.

ISSN: 0029-7844/16 significantly different in the study compared with the control group. In moderate and severe intrahepatic cholestasis of pregnancy, meconium staining was observed significantly more often compared with that in a control group $(23.0 \%$ and $32.3 \%$ compared with $11.4 \%, P<01)$. There was no correlation between estimated blood loss or $\Delta \mathrm{Hb}$ and severity of intrahepatic cholestasis of pregnancy.

CONCLUSIONS: In our cohort of women with intrahepatic cholestasis of pregnancy who are treated with ursodeoxycholic acid and have planned delivery (induction of labor or planned cesarean delivery) at 38 weeks of gestation, no differences in postpartum blood loss were seen.

(Obstet Gynecol 2016;0:1-5)

DOI: 10.1097/AOG.0000000000001693

Intrahepatic cholestasis of pregnancy is the most common liver condition uniquely manifested during pregnancy. ${ }^{1}$ The diagnosis of intrahepatic cholestasis of pregnancy is made by exclusion of other causes as well as the presence of clinical features in association with elevated bile acid levels. ${ }^{2}$ Intrahepatic cholestasis of pregnancy has been associated with a variety of adverse fetal outcomes, including preterm delivery, meconium staining of the amniotic fluid, and intrauterine fetal demise. ${ }^{3-5}$ Studies have shown a significant correlation of the level of serum bile acids with the incidence of adverse events. ${ }^{5,6}$

Apart from the occasionally massive distress caused by the generalized pruritus, intrahepatic cholestasis of pregnancy rarely poses a severe risk to maternal health; however, a rare and serious threat for the mother can occur in the presence of steatorrhea from fat malabsorption and subsequently reduced levels of vitamin K-dependent coagulation factors II, VII, IX, or $\mathrm{X}^{7}$ An increased risk for intrapartum hemorrhage induced by intrahepatic cholestasis of pregnancy has been suggested, ${ }^{8}$ especially in women who did not receive vitamin $\mathrm{K}$ in pregnancy. ${ }^{9,10}$ 
However, no difference in coagulation parameters (partial thromboplastin time, platelet count) in women with intrahepatic cholestasis of pregnancy was reported. ${ }^{11}$ Because the association of postpartum hemorrhage with intrahepatic cholestasis of pregnancy was not proven in a large study population, the objective of our study was to analyze postpartum blood loss in women with intrahepatic cholestasis of pregnancy and to evaluate the association of bile acid levels (severity of intrahepatic cholestasis of pregnancy) with postpartum blood loss.

\section{MATERIALS AND METHODS}

This is a retrospective, case-control, single-center study. Data were collected from our electronic database, Perinat, which contains all the patients' diagnoses and prospectively collected clinical data on the course of the pregnancy, delivery as well as maternal and neonatal outcome. Data were recorded prospectively immediately after the delivery by the midwife and the attending physician. The study has ethical approval according to the ethics review board of Zurich (reference number KEK-ZH-Nr. 2015-0449).

All deliveries after 22 weeks of gestation at our University Hospital from November 2004 to November 2014 were included. Exclusion criteria for both women in the case group and those in the control group were known risk factors for postpartum bleeding such as placentation pathology, known coagulation disorders, and liver dysfunction. The study examined 15,083 women including 348 women with intrahepatic cholestasis of pregnancy (2.3\%). All women with intrahepatic cholestasis of pregnancy were treated with $900 \mathrm{mg}$ ursodeoxycholic acid administered twice daily, which is the standard dosage at our institution, and were planned for induction of labor or planned cesarean delivery at 38 weeks of gestation. Women with intrahepatic cholestasis of pregnancy usually did not receive increased antenatal surveillance such as a nonstress test or biophysical profile before 38 weeks of gestation. The therapy regimen for intrahepatic cholestasis of pregnancy did not change throughout the study period.

Data on the following obstetric parameters were collected: cholestasis of pregnancy, bile acid level, number of fetuses, parity, induction of labor, maternal age at delivery, body mass index (BMI, calculated as weight $\left.(\mathrm{kg}) /[\text { height }(\mathrm{m})]^{2}\right)$, estimated blood loss, blood transfusion, mode of delivery (spontaneous deliveries, assisted vaginal deliveries, type of cesarean delivery [elective, unplanned, repeat], urgent cesarean delivery), hemoglobin $(\mathrm{Hb})$ level before and after delivery, and gestational age at birth. The obtained neonatal information included admission to the neonatal intensive care unit, neonatal birth weight, arterial umbilical cord $\mathrm{pH}$, 5-minute and 10-minute Apgar scores, meconium staining of the amniotic fluid, and peripartum demise.

To adjust for differences in baseline characteristics, a propensity analysis was performed and women in the control group were matched to the women in the intrahepatic cholestasis of pregnancy case group in a 5:1 ratio (1,725 compared with 345$)$. Propensity score matching was performed on a logit scale with a caliper of 0.2. During the matching process, three women with intrahepatic cholestasis of pregnancy revealed no propensity scores as a result of missing data and hence were excluded from further analysis. The following criteria were used for matching: gestational age, maternal age, maternal BMI, multiparity, multiple gestation, induction of labor, and mode of delivery (including 21 possibilities of spontaneous vaginal delivery, vaginal operative delivery, elective and unplanned cesarean delivery, and repeat cesarean delivery according to delivery mode and parity). Baseline comparison between women with and without intrahepatic cholestasis of pregnancy was calculated. Differences between these two groups were analyzed with Fisher exact test for categorical variables (multiple gestation, induction of labor, parity) and with the two-tailed $t$ test for numeric variables (BMI, maternal age, gestational age).

In a second step, the outcome of these two groups was compared pairwise with the Mann-Whitney $U$ test for continuous variables and the Fisher exact test for categorical variables. Blood loss was analyzed by estimated blood loss and $\Delta \mathrm{Hb}$ (difference between prepartum and postpartum $\mathrm{Hb}$ ). In our institution, determination of $\mathrm{Hb}$ level is performed routinely prepartum as well as postpartum for all parturient women.

Finally, three subgroups were defined in women with intrahepatic cholestasis of pregnancy regarding the severity of intrahepatic cholestasis of pregnancy using maximum level of bile acids (mild [less than 40 micromoles/L], moderate [40-99 micromoles/L], and severe intrahepatic cholestasis of pregnancy [100 micromoles/L or greater]). Differences in estimated blood loss, $\Delta \mathrm{Hb}$, and meconium staining of the amniotic fluid between subgroups were analyzed with $\chi^{2}$ test and Kruskal-Wallis test. A Spearman rank correlation was performed to evaluate the association of bile acid level and blood loss within subgroups.

In case of missing values, the respective patient was excluded from the related analysis. Regarding the analysis of blood loss, only four patients with intrahepatic cholestasis of pregnancy had to be excluded as 
a result of unreliable estimated blood loss. $\Delta \mathrm{Hb}$ was obtained in all patients with intrahepatic cholestasis of pregnancy. In the control group, 15 patients were excluded from analysis for differences in $\Delta \mathrm{Hb}$ as a result of incomplete data.

All statistical analyses were performed using IBM SPSS Statistics 22.0 and the R for propensity score matching. $P<.05$ was considered statistically significant.

\section{RESULTS}

After matching, all baseline characteristics were comparable between study and control groups (Table 1). Neonatal and maternal outcome is shown in Table 2. The estimated blood loss was not significantly higher in the intrahepatic cholestasis of pregnancy group. There was no difference in the $\mathrm{Hb}$ levels prepartum and postpartum between groups. The number of blood transfusions did not differ between the two groups. Meconium staining of amniotic fluid was not significantly more frequent in women with cholestasis. The number of stillbirths was not significantly different in the nonintrahepatic cholestasis of pregnancy group in stillbirths after 26 weeks of gestation. In women with intrahepatic cholestasis of pregnancy, two cases of stillbirth after 26 weeks of gestation were observed. One patient was admitted to the hospital with preterm prelabor rupture of membranes at 33 weeks of gestation and bile acid level of 96 micromoles/L. The second case was associated with a generalized nonimmunologic fetal hydrops at 29 weeks of gestation.
Neonatal outcome parameters were also comparable between the two groups: umbilical arterial $\mathrm{pH}$, the 5-minute Apgar score as well as the rate of admission to the neonatal care unit were similar in both groups. Ten-minute Apgar score was significantly higher in the intrahepatic cholestasis of pregnancy group, although clinically not relevant.

Among the 345 women with intrahepatic cholestasis of pregnancy, there were 240 cases of mild, 74 of moderate, and 31 of severe cholestasis. In the subgroup analysis, there was no significant association between bile acid levels and estimated blood loss or $\Delta \mathrm{Hb}$ (Table 3).

Meconium staining of the amniotic fluid correlated with the severity of intrahepatic cholestasis of pregnancy. In moderate and severe intrahepatic cholestasis of pregnancy, meconium staining was observed significantly more often compared with women in the control group.

\section{DISCUSSION}

Our results do not support the theory of increased postpartum blood loss resulting from intrahepatic cholestasis of pregnancy in our cohort of actively managed women. Estimated blood loss and $\Delta \mathrm{Hb}$ were not significantly higher in the intrahepatic cholestasis of pregnancy group.

Blood loss of more than $500 \mathrm{~mL}$ was found in one study in $17 \%$ of 70 women with intrahepatic cholestasis of pregnancy ${ }^{9}$ and in another study in $20 \%$ of 50 women with intrahepatic cholestasis of pregnancy. ${ }^{8}$ On the contrary, DeLeon ${ }^{11}$ found blood loss of more than $500 \mathrm{~mL}$ in women with intrahepatic cholestasis

Table 1. Baseline Characteristics After Matching

\begin{tabular}{|c|c|c|c|}
\hline Baseline Characteristics & $\begin{array}{l}\text { Intrahepatic Cholestasis } \\
\text { of Pregnancy }(n=345)\end{array}$ & $\begin{array}{l}\text { Women in the } \\
\text { Control Group }(n=1,725)\end{array}$ & $P$ \\
\hline Multiple gestation & $79(22.9)$ & $393(22.8)$ & $.94 *$ \\
\hline Induction of labor & $140(40.6)$ & $693(40.2)$ & $.90^{*}$ \\
\hline Planned cesarean delivery & $95(27.5)$ & $471(27.7)$ & $.98^{*}$ \\
\hline Unplanned cesarean delivery & $80(23.2)$ & $435(25.2)$ & $.47^{*}$ \\
\hline Spontaneous vaginal deliveries & $147(42.6)$ & $723(41.9)$ & $.86^{*}$ \\
\hline Operative vaginal deliveries & $23(6.7)$ & $96(5.6)$ & $.50 *$ \\
\hline Multiparity & $143(41.4)$ & $697(40.4)$ & $.72 *$ \\
\hline $\mathrm{BMI}\left(\mathrm{kg} / \mathrm{m}^{2}\right)$ & $22.9 \pm 16.8$ & $22.9 \pm 4.3$ & $.82^{+}$ \\
\hline Age $(y)$ & $32.0 \pm 5.7$ & $32.1 \pm 5.5$ & $.63^{+}$ \\
\hline Gestational age $(d)$ & $262.6 \pm 16.8$ & $262.1 \pm 25.7$ & $.75^{+}$ \\
\hline
\end{tabular}

BMI, body mass index.

Data are $\mathrm{n}(\%)$ or mean \pm standard deviation unless otherwise specified.

Matching criteria: gestational age, maternal age, maternal BMI, multiparity, multiple gestation, induction of labor, and mode of delivery (including 21 possibilities of spontaneous vaginal delivery, vaginal operative delivery, elective and unplanned cesarean delivery, and repeat cesarean delivery according to delivery mode and parity).

* Fisher exact test.

+ Two-tailed $t$ test. 
Table 2. Neonatal and Maternal Outcomes in Women With Intrahepatic Cholestasis of Pregnancy and Women in the Control Group

\begin{tabular}{|c|c|c|c|}
\hline Outcome & $\begin{array}{l}\text { Intrahepatic Cholestasis } \\
\text { of Pregnancy }(n=345)\end{array}$ & $\begin{array}{l}\text { Women in the } \\
\text { Control Group }(n=1,725)\end{array}$ & $P$ \\
\hline \multicolumn{4}{|l|}{ Maternal } \\
\hline $\mathrm{EBL}(\mathrm{mL})$ & $400(300-600)$ & $400(300-600)$ & $.22^{*}$ \\
\hline$\Delta \mathrm{Hb}(\mathrm{g} / \mathrm{L})$ & $14.0(5-22)$ & $12.0(4-21)$ & $.09^{*}$ \\
\hline Blood transfusion & $3(0.9)$ & $10(0.6)$ & $.47^{+}$ \\
\hline \multicolumn{4}{|l|}{ Neonatal } \\
\hline Birth weight (g) & $3,060(2,595-3,465)$ & $3,030(2,420-3,490)$ & $.37^{*}$ \\
\hline Meconium staining & $50(14.5)$ & $197(11.4)$ & $.12^{+}$ \\
\hline Stillbirth & $3(0.9)$ & $62(3.6)$ & $<.01^{\dagger}$ \\
\hline Stillbirth at greater than $26 \mathrm{wk}$ of gestation & $2(0.6)$ & $31(1.8)$ & $.10^{\dagger}$ \\
\hline Umbilical arterial $\mathrm{pH}$ & $7.28 \pm 0.07$ & $7.29 \pm 0.19$ & $<.01^{*}$ \\
\hline 5-min Apgar score & $9(9-9)$ & $9(9-9)$ & $.37^{*}$ \\
\hline 10-min Apgar score & $9(9-10)$ & $9(9-9)$ & $<.01^{*}$ \\
\hline Admission to neonatal care unit & $55(15.9)$ & $290(16.8)$ & $.75^{+}$ \\
\hline
\end{tabular}

$\mathrm{EBL}$, estimated blood loss; $\Delta \mathrm{Hb}$, difference between prepartum and postpartum hemoglobin.

Data are median (25th percentile-75th percentile), n (\%), or mean \pm standard deviation unless otherwise specified.

* Mann-Whitney $U$ test.

${ }^{+}$Fisher exact test.

of pregnancy in only $2.4 \%$ after vaginal delivery and $6.3 \%$ after cesarean delivery. Brouwers et $\mathrm{al}^{6}$ defined postpartum hemorrhage as a blood loss of more than $1,000 \mathrm{~mL}$, which was found in $7.4 \%$ of 215 patients. However, in these studies, no comparison with a control group was performed. Furthermore, analysis of blood loss was done by estimation of blood loss, whereas our study also calculated $\Delta \mathrm{Hb}$ as an objective parameter.

The role of vitamin $\mathrm{K}$ in women with intrahepatic cholestasis of pregnancy is still unclear. In a small study of 70 women including 11 women without vitamin $\mathrm{K}$ supplementation, Kenyon et $\mathrm{al}^{9}$ found that postpartum bleeding was increased. Only one national guideline deals with intrahepatic cholestasis of pregnancy and a possible benefit from vitamin $\mathrm{K}$ supplementation in the presence of malabsorption. ${ }^{10}$ Contrary to the recommendation of the Royal
College of Obstetricians and Gynaecologists, we did not routinely measure prothrombin time in women with intrahepatic cholestasis of pregnancy throughout pregnancy and vitamin $\mathrm{K}$ was not administered before delivery. In accordance with the results of our study, the role of vitamin $\mathrm{K}$ in postpartum bleeding in women with intrahepatic cholestasis of pregnancy might be questioned.

In our cohort of 345 women with intrahepatic cholestasis of pregnancy who followed a standard therapy regimen toward active management for intrahepatic cholestasis of pregnancy, first by treating every patient with $900 \mathrm{mg}$ ursodeoxycholic acid twice a day and second by planned delivery after 38 completed weeks of gestation, no increased incidence of adverse fetomaternal outcome was observed apart from increased meconium staining in the subgroup of women with severe intrahepatic cholestasis of pregnancy. Induction of labor was

Table 3. Incidence of Meconium, Delta Hemoglobin, and Estimated Blood Loss in the Subgroups of Intrahepatic Cholestasis of Pregnancy

\begin{tabular}{|c|c|c|c|c|c|}
\hline Outcome & $\begin{array}{l}\text { Women in the Control } \\
\text { Group }(n=1,725)\end{array}$ & $\begin{array}{c}\text { Mild } \\
(n=240)\end{array}$ & $\begin{array}{l}\text { Moderate } \\
(n=74)\end{array}$ & $\begin{array}{l}\text { Severe } \\
(\mathrm{n}=31)\end{array}$ & $P$ \\
\hline Meconium staining & 11.4 & 9.6 & 23 & 32.3 & $<.01 *$ \\
\hline$\Delta \mathrm{Hb}(\mathrm{g} / \mathrm{L})$ & $12(12-21)$ & $13.5(13.5-22)$ & $13.5(13.5-22)$ & $17(17-24)$ & $.26^{\dagger}$ \\
\hline $\mathrm{EBL}(\mathrm{mL})$ & $400(300-600)$ & $400(300-600)$ & $500(300-625)$ & $450(300-500)$ & $.37^{+}$ \\
\hline
\end{tabular}

$\Delta \mathrm{Hb}$, difference between prepartum and postpartum hemoglobin; $\mathrm{EBL}$, estimated blood loss.

Data are \% or median (25th percentile-75th percentile) unless otherwise specified.

Mild intrahepatic cholestasis of pregnancy, less than 40 micromoles/L bile acid; moderate intrahepatic cholestasis of pregnancy, 40-99 micromoles/L bile acid; severe intrahepatic cholestasis of pregnancy, 100 micromoles/L or greater bile acid.

$* \chi^{2}$ test.

+ Kruskal-Wallis test. 
performed in 40\% of patients with intrahepatic cholestasis of pregnancy. We used a relatively high dose of ursodeoxycholic acid (1,800 mg daily) compared with most other studies. ${ }^{12,13}$ This treatment protocol was based on an interdisciplinary consensus, the reasonable pharmacologic safety of ursodeoxycholic acid, and reported dosages of up to $2 \mathrm{~g}^{14}$

In our cohort, there were only two stillbirths in women with intrahepatic cholestasis of pregnancy after 26 weeks of gestation. This small number prevents meaningful comparison with our control group. Neonatal outcome was not the focus of our study, but these results, in light of data suggesting a higher stillbirth rate in women with intrahepatic cholestasis of pregnancy, encourage us to continue our current active management of patients with intrahepatic cholestasis of pregnancy.

The strength of our study is that we analyzed blood loss with two different methods, first by calculation of $\Delta \mathrm{Hb}$ and second by measurement of blood loss during delivery. Blood loss is measured in all vaginal deliveries at our institution with a quantitative method. In case of increased bleeding, which is verified by regularly weighing the pads, a plastic bag with a quantitative scale is installed under the woman's pelvis. It is known that without a quantitative measurement method, blood loss is often underestimated. ${ }^{15,16}$ The calculation of $\Delta \mathrm{Hb}$ by measurement before and after delivery poses an objective variable to examine blood loss during birth. Because it is routine in our institution to determine $\mathrm{Hb}$ levels in all parturient women before as well as after delivery, no woman with intrahepatic cholestasis of pregnancy and only 15 women in the control group were excluded from analysis as a result of missing $\Delta \mathrm{Hb}$. Moreover, matching was possible for all relevant criteria (multiple gestation, induction of labor, parity, maternal age, gestational age, BMI) in our study population with 15,083 women. Without matching, no appropriate comparison is possible, because the frequency of multiple gestation and induction of labor is higher as a result of the pathophysiology and management of intrahepatic cholestasis of pregnancy. This statistical approach is an important advantage of our study. Apart from possible limitations of a single-center study, its positive aspect was that a uniform therapy management was applied over the entire study period.

In conclusion, according to our data, women with intrahepatic cholestasis of pregnancy do not show increased postpartum blood loss or significant adverse outcomes in pregnancies with an active management strategy including $2 \times 900 \mathrm{mg}$ ursodeoxycholic acid daily and planned delivery by induction of labor or planned cesarean delivery at 38 weeks of gestation. Administration of vitamin $\mathrm{K}$ does not appear to be necessary.

\section{REFERENCES}

1. Diken Z, Usta IM, Nassar AH. A clinical approach to intrahepatic cholestasis of pregnancy. Am J Perinatol 2014;31:1-8.

2. Walker IA, Nelson-Piercy C, Williamson C. Role of bile acid measurement in pregnancy. Ann Clin Biochem 2002;39: 105-13.

3. Geenes V, Williamson C. Intrahepatic cholestasis of pregnancy. World J Gastroenterol 2009;15:2049-66.

4. Puljic A, Kim E, Page J, Esakoff T, Shaffer B, LaCoursiere DY, et al. The risk of infant and fetal death by each additional week of expectant management in intrahepatic cholestasis of pregnancy by gestational age. Am J Obstet Gynecol 2015;212: 667.e1-5.

5. Glantz A, Marschall HU, Mattsson LA. Intrahepatic cholestasis of pregnancy: relationships between bile acid levels and fetal complication rates. Hepatology 2004;40:467-74.

6. Brouwers L, Koster M, Page-Christiaens G, Kemperman H, Boon J, Evers IM, et al. Intrahepatic cholestasis of pregnancy: maternal and fetal outcomes associated with elevated bile acid levels. Am J Obstet Gynecol 2015;212:100.e1-7.

7. Horlocker TT, Wedel DJ, Rowlingson JC, Enneking FK, Kopp SL, Benzon HT, et al. Regional anesthesia in the patient receiving antithrombotic or thrombolytic therapy: American Society of Regional Anesthesia and Pain Medicine Evidence-Based Guidelines (Third Edition). Reg Anesth Pain Med 2010;35: 64-101.

8. Reid R, Ivey KJ, Rencoret RH, Storey B. Fetal complications of obstetric cholestasis. Br Med J 1976;1:870-2.

9. Kenyon AP, Piercy CN, Girling J, Williamson C, Tribe RM, Shennan AH. Obstetric cholestasis, outcome with active management: a series of 70 cases. BJOG 2002;109:282-8.

10. Royal College of Obstetricians \& Gynaecologists. Obstetric cholestasis. Green-top Guideline No. 43. London (United Kingdom): RCOG; 2011.

11. DeLeon A, De Oliveira GS, Kalayil M, Narang S, McCarthy RJ, Wong CA. The incidence of coagulopathy in pregnant patients with intrahepatic cholestasis: should we delay or avoid neuraxial analgesia? J Clin Anesth 2014;26:623-7.

12. Chappell LC, Gurung V, Seed PT, Chambers J, Williamson C, Thornton JG, et al. Ursodeoxycholic acid versus placebo, and early term delivery versus expectant management, in women with intrahepatic cholestasis of pregnancy: semifactorial randomised clinical trial. BMJ 2012;344:e3799.

13. Bacq Y, Sentilhes L, Reyes H, Glantz A, Kondrackiene J, Binder $\mathrm{T}$, et al. Efficacy of ursodeoxycholic acid in treating intrahepatic cholestasis of pregnancy: a meta-analysis. Gastroenterology 2012;143:1492-501.

14. Williamson C, Geenes V. Intrahepatic cholestasis of pregnancy. Obstet Gynecol 2014;124:120-33.

15. Dildy GA 3rd, Paine AR, George NC, Velasco C. Estimating blood loss: can teaching significantly improve visual estimation? Obstet Gynecol 2004;104:601-6.

16. Yoong W, Karavolos S, Damodaram M, Madgwick K, Milestone N, Al-Habib A, et al. Observer accuracy and reproducibility of visual estimation of blood loss in obstetrics: how accurate and consistent are health-care professionals? Arch Gynecol Obstet 2010;281:207-13. 